\title{
The Evolving Role of Radiotherapy in Locally Advanced Rectal Cancer and the Potential for Nonoperative Management
}

\author{
Karishma Khullar, ${ }^{1}$ Nell Maloney Patel, ${ }^{2}$ Cristan Anderson, ${ }^{2}$ Anupama Chundury, ${ }_{1}^{1}$ Darren Carpizo, ${ }^{3}$ Daniel Feingold, ${ }^{3}$ \\ Miral Grandhi, ${ }^{3}$ Howard Hochster, ${ }^{4}$ Krupa Jani, ${ }^{1}$ Timothy Kennedy, ${ }^{3}$ Russell Langan, ${ }^{3}$ Kristen Spencer, ${ }^{4}$ David August, ${ }^{2}$ \\ and Salma K Jabbour \\ 1. Department of Radiation Oncology, Rutgers Cancer Institute of New Jersey, Rutgers University, New Brunswick, NJ, USA; 2. Department of Surgery, \\ Rutgers Robert Wood Johnson Medical School, Rutgers University, New Brunswick, NJ, USA; 3. Division of Surgical Oncology, Rutgers Cancer Institute \\ of New Jersey, Rutgers Robert Wood Johnson Medical School, Rutgers University, New Brunswick, NJ, USA; 4. Division of Medical Oncology, Rutgers \\ Cancer Institute of New Jersey, Rutgers University, New Brunswick, NJ, USA
}

DOl: https://doi.org/10.17925/OHR.2020.16.1.43 ocally advanced rectal cancer has broadly been defined as T3, T4, or lymph node-positive disease. In the 1990s, adjuvant chemoradiation was considered the optimal management for locally advanced rectal cancer. However, the paradigm shifted when the German CAO/ARO/AIO-94 Rectal Cancer trial established neoadjuvant chemoradiation as the standard of care, based on reduced rates of toxicity and local recurrence, as well as higher rates of sphincter preservation compared with postoperative chemoradiation. Both short-course radiation and long-course chemoradiation are currently accepted methods for neoadjuvant treatment, with recent trials showing equivalence in outcomes. While surgery remains the cornerstone of treatment, there are data supporting the use of magnetic resonance imaging for risk stratification in rectal cancer and encouraging prospective data regarding nonoperative management. This review summarizes data on the evolution of treatment for locally advanced rectal cancer and discusses emerging evidence for nonoperative management.

\section{Keywords}

Rectal cancer, chemotherapy, radiotherapy, watchful waiting, neoadjuvant therapy, nonoperative management, chemoradiation

Disclosures: Karishma Khullar, Nell Maloney Patel, Cristan Anderson, Anupama Chundury, Darren Carpizo, Daniel Feingold, Miral Grandhi, Howard Hochster, Krupa Jani, Timothy Kennedy, Russell Langan, Kristen Spencer, David August, and Salma K Jabbour have no financial or non-financial relationships or activities to declare in relation to this article. This work was carried out with the help of Grant Funding, R01CA200800.

Review Process: Double-blind peer review.

Compliance with Ethics: This article involves a review of the literature and does not report on new clinical data or any studies with human or animal subjects performed by any of the authors.

Authorship: All named authors meet the criteria of the International Committee of Medical Journal Editors (ICMJE) for authorship for this manuscript, take responsibility for the integrity of the work as a whole and have given final approval for the version to be published.

Access: This article is freely accessible at touchONCOLOGY.com (c) Touch Medical Media 2020.

Received: December 11, 2019

Accepted: January 29, 2020

Published Online: April 28, 2020

Citation: Oncology \& Hematology Review (US).

2020:16(1):43-51

Corresponding Author: Salma K Jabbour, Rutgers Cancer Institute of New Jersey, 195 Little Albany Street, New Brunswick, NJ 08903, USA. E: Jabbousk@cinj.rutgers.edu

Support: No funding was received for the publication of this article.
Colorectal cancer is the third most common cancer worldwide and the fourth most common cause of death, with 1.8 million new cases and 881,000 deaths in 2018. ${ }^{1}$ Rectal cancers account for $28 \%$ of these cancers. ${ }^{2}$ Most colorectal cancers arise from adenomatous polyps and progress to adenocarcinoma through the three major genetic pathways of chromosomal instability, microsatellite instability, and CpG island methylation. ${ }^{3}$ Locally advanced rectal cancer is often defined as T3, T4, or lymph node-positive disease, and the treatment paradigm has evolved over time from surgery alone, which resulted in high rates of locoregional recurrences, particularly in the absence of total mesorectal excision (TME), ${ }^{4-6}$ to the current standard of care-neoadjuvant chemoradiotherapy (CRT) followed by TME. ${ }^{7.8}$ While surgery remains the main component for the curative treatment for locally advanced rectal cancer, nonoperative management is a strategy that is currently being investigated in prospective trials. The purpose of this review is to summarize data on the evolution of management for locally advanced rectal cancer, as well as to comment upon emerging evidence for nonoperative management.

\section{Early treatment for rectal cancer}

Developed in 1908, the first effective treatment for rectal cancer was the abdominoperineal resection (APR). ${ }^{9}$ Although procedural mortality rates were high, the operation not only addressed the need to remove the primary rectal cancer, but also recognized the importance of regional node drainage. subsequent technical refinements in the procedure, and improved perioperative care, reduced both operative morbidity and mortality. With the recognition that lymphatic drainage from rectal cancers was cephalad and progressed retrograde to the perineum only when bulky tumors obstructed proximal lymphatic flow, the sphincter-sparing procedure known as low anterior resection (LAR) became more common. ${ }^{10}$ More recently, an emphasis on TME has improved locoregional disease control rates significantly due to en bloc resection of the mesorectum, which contains the perirectal lymph nodes, the first echelon of rectal lymph node drainage. ${ }^{11}$

Moving into the 1990s, the primary modality for rectal cancer treatment was surgery followed by adjuvant radiotherapy (RT). Several randomized trials have examined this treatment approach, as summarized in Table 1. For example, in the Gastrointestinal Study Group prospective trial, patients who had undergone either LAR or APR for locally advanced rectal adenocarcinoma were randomized to 
Table 1: Early adjuvant chemoradiation trials for rectal cancer

\begin{tabular}{|c|c|c|c|c|c|}
\hline Author & Year & Patient population & Surgical resection? & Study arms & Results \\
\hline $\begin{array}{l}\text { Gastrointestinal Tumor } \\
\text { Study Group }{ }^{12}\end{array}$ & 1985 & $\begin{array}{l}227 \text { patients who } \\
\text { underwent curative } \\
\text { surgical resection for } \\
\text { rectal adenocarcinoma }\end{array}$ & Yes_LAR or APR & $\begin{array}{l}\text { 1. No adjuvant treatment ( } n=58 \text { ) } \\
\text { 2. Postoperative RT to a dose of } 4,000 \\
\text { or } 4,800 \text { rad ( } n=50) \\
\text { 3. Postoperative chemotherapy with } \\
5 \text {-FU and semustine (methyl-CCNU) } \\
\text { ( } n=48) \\
\text { 4. Combination chemoradiation } \\
(n=46)\end{array}$ & $\begin{array}{l}\text { - Median time to recurrence in control } \\
\text { arm was } 31 \text { months and was not } \\
\text { reached in the other arms } \\
\text { - } \text { Recurrence rates by arm: } \\
55 \% \text { controls, } 46 \% \text { chemotherapy } \\
\text { arm, } 48 \% \text { RT arm, and } \\
33 \% \text { combination arm } \\
\text { - } \text { Time to tumor recurrence differed } \\
\text { significantly among groups ( } p=0.04 \text { ) } \\
\text { - } \text { Comparison of combined modality } \\
\text { versus resection alone favored } \\
\text { combined modality ( } p=0.009 \text { ) } \\
\text { - } \text { comparison of combined modality } \\
\text { versus RT or combined modality } \\
\text { versus chemotherapy were not } \\
\text { significant ( } p=0.16 \text { ) } \\
\text { - No significant interaction between } \\
\text { chemotherapy and RT ( } p>0.42 \text { ), } \\
\text { indicating separate and additive } \\
\text { effects of both treatments } \\
\text { - Overall survival did not differ } \\
\text { significantly between groups ( } p=0.20 \text { ) }\end{array}$ \\
\hline NSABP R-0113 & 1988 & $\begin{array}{l}555 \text { patients with } \\
\text { Dukes' B and C rectal } \\
\text { cancers treated with } \\
\text { curative resection }\end{array}$ & Yes-LAR or APR & $\begin{array}{l}\text { 1. No further treatment ( } n=184 \text { ) } \\
\text { 2. Postoperative chemotherapy with } \\
5-\mathrm{FU} \text {, semustine, and vincristine } \\
\text { ( } \mathrm{n}=187 \text { ) } \\
\text { 3. Postoperative RT to a dose of } \\
4,600-4,700 \mathrm{rad} \text { with boost to } \\
5,100-5,300 \mathrm{rad}(\mathrm{n}=184)\end{array}$ & $\begin{array}{l}\text { - The chemotherapy group } \\
\text { demonstrated a significant } \\
\text { disease-free survival ( } p=0.006 \text { ) and } \\
\text { overall survival benefit ( } p=0.05) \\
\text { - The RT demonstrated a trend } \\
\text { towards improvement in locoregional } \\
\text { recurrence }(25 \% \text { versus } 16 \%, p=0.06 \text { ), } \\
\text { but no difference in disease-free } \\
\text { survival ( } p=0.4) \text { or overall survival } \\
(p=0.7)\end{array}$ \\
\hline $\begin{array}{l}\text { Medical Research } \\
\text { Council Rectal Cancer } \\
\text { Working Party }\end{array}$ & 1996 & $\begin{array}{l}469 \text { patients with } \\
\text { mobile Dukes' stage B } \\
\text { and } \mathrm{C} \text { rectal cancers } \\
\text { who underwent } \\
\text { curative resection }\end{array}$ & Yes-LAR or APR & $\begin{array}{l}\text { 1. Surgery alone } \\
\text { 2. Surgery followed by RT to a dose of } \\
40 \text { Gy in } 20 \text { fractions }\end{array}$ & $\begin{array}{l}\text { RT decreased local recurrence as } \\
\text { compared with surgery alone } \\
(21 \% \text { versus } 34 \%, p=0.001) \\
\text { - No significant difference in overall } \\
\text { survival ( } p=0.17) \text {, distant recurrence } \\
(p=0.18) \text {, and disease-free survival } \\
(p=0.18)\end{array}$ \\
\hline
\end{tabular}

5-FU =5-fluorouracil; $A P R=$ abdominoperineal resection; $G y=$ gray, unit of absorbed radiation dose; $L A R=$ low anterior resection; methyl-CCNU = methyl-chloroethyl cyclohexyl nitrosourea; rad = radiation absorbed dose; $R T$ = radiotherapy.

receive no adjuvant therapy, postoperative RT, postoperative chemotherapy, or a combination of postoperative CRT. ${ }^{12}$ With a median follow-up of 80 months, overall survival (OS) was not significantly different among the treatment groups $(p=0.20)$, but the local recurrence (LR) rate was highest in the patients who received no adjuvant therapy and lowest in those who received adjuvant CRT (55\% versus 33\%). ${ }^{12}$ The median time to recurrence in the control arm that received no adjuvant therapy was 31 months, and the median time to recurrence was not reached in the other arms. Nevertheless, the time to tumor recurrence differed significantly among the four treatment groups $(p<0.04)$ and was significantly prolonged by combined CRT as compared with resection alone $(p<0.009) .{ }^{12}$ It is important to note, however, that TME was not routinely performed at the time of this seminal study. Thus, this study may have resulted in higher LR rates than could be achieved with TME-based operations.
In the National Surgical Adjuvant Breast and Bowel Project (NSABP) R-01 protocol, 555 patients with Dukes' B and C rectal cancers treated by either LAR or APR were randomized to no further treatment, postoperative adjuvant chemotherapy or postoperative RT (Table 1). ${ }^{13}$ When compared with surgery alone, the chemotherapy group had improved disease-free survival (DFS) and $\mathrm{OS}$ ( $p=0.006$ and $p=0.05$, respectively), though the benefit appeared to be restricted to males and younger patients. ${ }^{13}$ When the radiation group was compared with surgery alone, RT showed a trend towards improved locoregional control (16\% versus $25 \%$, respectively for locoregional recurrence, $p=0.06$ ), but no difference in DFS or OS. ${ }^{13}$

The Medical Research Council Rectal Cancer Working Party randomized 469 patients to surgery alone by either LAR or APR versus surgery, followed by adjuvant RT to a dose of 40 Gy in 20 fractions. ${ }^{14}$ 
At 5 years, adjuvant RT decreased LR as compared with surgery alone ( $21 \%$ versus $34 \%, p=0.001$ ), without any significant increase in serious late bowel complications, but there were no significant differences in OS, distant recurrence, and DFS. ${ }^{14}$ In summary, these trials demonstrated that while postoperative RT may improve local control, adjuvant chemotherapy may provide a survival benefit.

\section{Postoperative chemoradiotherapy}

In the 1990s, the National Cancer Institute Consensus Conference concluded that the standard postoperative treatment for patients with rectal cancer with pT3 or N1-2 disease should be CRT. ${ }^{15}$ Several subsequent trials shaped both type and route of chemotherapy based on providing the optimal benefit. In the US GI Intergroup 86-47-51 trial, 660 patients with stage II or III rectal cancer who had undergone curative surgery with either LAR or APR were randomized to four arms, as shown in Table 2. ${ }^{16}$ With a median follow-up of 46 months, patients who received protracted venous infusion 5 -fluorouracil (PVI 5-FU; continuous infusion) had a significant decrease in the overall rate of local tumor relapse from 47 to $37 \%(p=0.01)$ and distant metastasis from 40 to $31 \%$ as compared with patients who received bolus 5 - FU ( $p=0.03)$. Additionally, there was no benefit in patients who received semustine (methyl-chloroethyl cyclohexyl nitrosourea) plus 5-FU. Patients who received a PVI of 5-FU also had a significant improvement in the time to relapse from 53 to $63 \%$ at 4 years ( $p=0.01)$ and an improvement in OS from 60 to $79 \%$ ( $(\mathrm{p}=0.0005){ }^{16}$ This study has shaped the modern standard of care in giving radiosensitizing continuous infusion 5-FU during RT.

The successor trial, Intergroup (INT)-0114 was a four-arm trial in which 1,695 patients with $\mathrm{T} 3 / 4$ or node-positive rectal cancer were randomized to the following groups: (1) RT and bolus 5-FU, (2) RT and bolus 5-FU with leucovorin, (3) RT and bolus 5-FU with levamisole, or (4) RT and bolus 5-FU with leucovorin and levamisole, as shown in Table 2. ${ }^{17}$ With a median follow-up of 7.4 years, there was no difference in OS or DFS by drug regimen. ${ }^{17}$ However, patients with high-risk T3 node-positive or T4NO disease had a lower survival rate (45\%) compared with low-risk T1-2 node-positive or T3NO disease (70\%). LR did not vary by treatment type, but low-risk patients had a failure rate of $9 \%$ compared with 18\% for high-risk patients at 5 years $(p<0.0001) .^{77}$

Finally, the INT-0144 trial was a three-arm trial in which 1,917 patients were randomized to either: (1) bolus 5-FU in two cycles, before and after RT, to a dose of 45 Gy with a minimum boost of 5.4 Gy with concurrent PVI 5-FU; (2) PVI 5-FU before, after, and during RT; or (3) bolus 5-FU-leucovorin-levamisole before, after, and during RT. ${ }^{18}$ With a median follow-up of 5.7 years, there was no difference in OS or DFS among the groups and locoregional recurrence was low. However, grade 3 or grade 4 hematologic toxicity was significantly lower in the PVI arm when compared with any other arms of the study (4\% versus $49-55 \%$ ). In summary, these trials demonstrated that PVI 5-FU was the optimal choice for concurrent chemotherapy with postoperative RT in patients with rectal cancer.

\section{Preoperative radiation versus preoperative chemoradiotherapy}

Two major trials established the superiority of preoperative CRT over preoperative RT alone. In a trial by Gérard et al., 733 patients with resectable, locally advanced rectal cancer were randomized to either preoperative RT alone at a dose of $45 \mathrm{~Gy}$, or preoperative CRT with bolus
5-FU and leucovorin during weeks 1 and 5, with surgery planned 3-10 weeks afterwards. In both arms, $50 \%$ of patients received adjuvant 5-FU. ${ }^{19}$ While acute toxicity was greater in the CRT arm (14.6\% versus $2.7 \%$; $p<0.05)$, LR was lower with CRT ( $8.1 \%$ versus $16.5 \% ; p<0.05)$. There was no difference in sphincter preservation or 5-year OS. ${ }^{19}$ Additionally, EORTC 22921 randomized 1,011 patients with clinical stage T3 or T4 resectable rectal cancer to preoperative RT to a dose of $45 \mathrm{~Gy}$, preoperative CRT to a dose of $45 \mathrm{~Gy}$ with two cycles of 5-FU and leucovorin, preoperative RT and four cycles of postoperative chemotherapy; or preoperative CRT and postoperative chemotherapy. ${ }^{20}$ The compliance rates for preoperative chemotherapy were $82.0 \%$ versus $42.9 \%$ for postoperative chemotherapy. The 5-year OS for all four groups was $65.2 \%$ and there was no significant difference between the groups that received chemotherapy preoperatively or postoperatively $(p=0.84$ and $p=0.12$, respectively). ${ }^{20}$ However, the 5 -year LR rates were $8.7 \%, 9.6 \%$ and $7.6 \%$ in the groups that received chemotherapy preoperatively, postoperatively, or both preoperatively and postoperatively, respectively, versus $17.1 \%$ in the group that did not receive chemotherapy $(\mathrm{p}=0.002) .{ }^{20}$ Thus, in both of these trials, the addition of chemotherapy to radiation significantly improved local control rates.

\section{Preoperative versus postoperative chemoradiotherapy}

The seminal German CAO/ARO/AIO-94 Rectal Cancer Study Group established preoperative CRT as the current standard for treating locally advanced rectal cancer. As shown in Supplementary Figure 1, 827 patients with $\mathrm{T} 3, \mathrm{~T} 4$, or node-positive disease were randomized to neoadjuvant CRT followed by TME, or TME followed by adjuvant CRT, with bolus 5-FU rather than PVI 5-FU. Preoperative CRT not only decreased LR at 10 years ( $7 \%$ versus $10 \%, p=0.048)$, but also increased the likelihood of sphincter-preservation surgery in those initially felt to require an APR $39 \%$ versus $19 \%, p=0.004)$. Moreover, preoperative CRT decreased the incidence of acute grade $3-4$ toxicity ( $27 \%$ versus $40 \%, p=0.001$ ) and late grade $3-4$ toxicity ( $14 \%$ versus $24 \%, p=0.01)$. However, there were no differences in distant recurrence, OS or DFS. ${ }^{8}$

The NSABP R-03 trial also evaluated preoperative versus postoperative CRT for patients with locally advanced rectal cancer (Supplementary Figure 2). The 5-year DFS was significantly better in the preoperative CRT group $(64.7 \%$ versus $53.4 \%, \mathrm{p}=0.011)$ and $\mathrm{OS}$ trended towards significance (74.5\% versus $65.6 \%, \mathrm{p}=0.065)$. Among patients who received preoperative therapy, $15 \%$ achieved a pathologic complete response (PCR), none of which developed recurrence at last follow-up. Of note, the study accrued only 267 of the 900 planned patients, thereby limiting the statistical power to detect differences. ${ }^{21}$

Finally, the UK Medical Research Council evaluated short-course preoperative RT versus initial surgery with selective postoperative CRT only in patients with involvement of the circumferential resection margin (CRM; supplementary Figure 3). ${ }^{22}$ With a median follow-up of 4 years, patients who received preoperative therapy compared with selective postoperative treatment had significantly lower 3 -year LR rates (4.4\% versus $10.6 \%$, $\mathrm{p}<0.0001)$ and higher 3 -year DFS (77.5\% versus $71.5 \%, \mathrm{p}=0.013)$, but there was no difference in OS. ${ }^{22}$ The aforementioned trials provide substantial evidence favoring preoperative CRT in patients with locally advanced rectal cancer. 
Table 2: Postoperative chemoradiation trials for rectal cancer

\begin{tabular}{|c|c|c|c|c|c|}
\hline Author & Year & $\begin{array}{l}\text { Patient } \\
\text { population }\end{array}$ & $\begin{array}{l}\text { Surgical } \\
\text { resection? }\end{array}$ & Study arms & Results \\
\hline O'Connell et al. ${ }^{16}$ & 1994 & $\begin{array}{l}660 \text { patients } \\
\text { with stage II or III } \\
\text { rectal cancer after } \\
\text { curative surgery }\end{array}$ & Yes-LAR or APR & $\begin{array}{l}\text { 1. Systemic chemotherapy with } \\
\text { 5-FU and semustine, plus RT to a } \\
\text { dose of } 45 \text { Gy with a minimum boost of } \\
5.4 \text { Gy and concomitant bolus injection } \\
\text { of 5-FU } \\
\text { 2. Systemic chemotherapy with 5-FU and } \\
\text { semustine, plus RT and concomitant } \\
\text { PVI 5-FU } \\
\text { 3. Systemic chemotherapy with 5-FU, plus } \\
\text { RT and concomitant bolus injection } \\
\text { of 5-FU } \\
\text { 4. Systemic chemotherapy with 5-FU, plus } \\
\text { RT and concomitant PVI 5-FU }\end{array}$ & $\begin{array}{l}\text { - There was a significant decrease in overall rate } \\
\text { of tumor relapse in patients who received PVI } \\
\text { 5-FU (from } 47 \% \text { to } 37 \% \text { ) as compared with those } \\
\text { who received bolus injections during RT ( } p=0.01 \text { ) } \\
\text { - There was a significant decrease in distant } \\
\text { metastasis in patients who received PVI 5-FU } \\
\text { (from 40\% to 31\%) as compared with those who } \\
\text { received bolus injections during RT ( } p=0.03 \text { ) } \\
\text { - Patients who received a PVI 5-FU had a } \\
\text { significant improvement in the time to relapse } \\
\text { ( } p=0.01 \text { ) and survival ( } p=0.005 \text { ), as compared } \\
\text { with those who received a bolus injection } \\
\text { of 5-FU. The time to relapse at } 4 \text { years was } \\
\text { increased from } 53 \% \text { to } 63 \% \text { and survival } \\
\text { increased from } 60 \% \text { to } 70 \% \text { among patients who } \\
\text { received a PVI 5-FU } \\
\text { There was no evidence of a beneficial effect in } \\
\text { the patients who received semustine plus 5-FU }\end{array}$ \\
\hline Tepper et al. ${ }^{17}$ & 2002 & $\begin{array}{l}\text { 1,695 patients with } \\
\text { T3/4 and } \\
\text { node-positive } \\
\text { rectal cancer }\end{array}$ & Yes-LAR or APR & $\begin{array}{l}\text { 1. Two cycles of 5-FU followed by } \\
\text { concurrent chemoradiation with 5-FU } \\
\text { to a dose of } 45.0 \text { Gy with a boost to } \\
\text { 50.0-50.4 Gy followed by two additional } \\
\text { cycles of 5-FU } \\
\text { 2. Two cycles of 5-FU and leucovorin } \\
\text { followed by concurrent chemoradiation } \\
\text { with 5-FU and leucovorin to a dose of } \\
\text { 45.0 Gy with a boost to 50.0-50.4 Gy } \\
\text { followed by two additional cycles of } \\
\text { 5-FU and leucovorin } \\
\text { 3. Two cycles of 5-FU and levamisole for } \\
\text { four cycles followed by concurrent } \\
\text { chemoradiation with 5-FU to a dose of } \\
45.0 \text { Gy with a boost to 50.0-50.4 Gy } \\
\text { followed by two additional cycles of } \\
\text { 5-FU and four cycles of levamisole } \\
\text { 4. Two cycles of 5-FU and leucovorin with } \\
\text { levamisole for four cycles followed } \\
\text { by concurrent CRT with 5-FU and } \\
\text { leucovorin to a dose of } 45.0 \text { Gy with a } \\
\text { boost to } 50.0-50.4 \text { Gy followed by two } \\
\text { additional cycles of 5-FU and leucovorin } \\
\text { and four cycles of levamisole }\end{array}$ & $\begin{array}{l}\text { - There was no difference in overall survival or } \\
\text { disease-free survival by drug regimen } \\
\text { - Patients with high-risk T3 node-positive or } \\
\text { T4N0 disease had a lower survival rate (45\%) } \\
\text { compared with low-risk T1-2 node-positive or } \\
\text { T3N0 disease (70\%) at } 7 \text { years } \\
\text { - Local recurrence did not vary by treatment } \\
\text { type, but low-risk patients had a failure rate of } \\
9 \% \text { compared with } 18 \% \text { for high-risk patients at } \\
5 \text { years }(p<0.0001)\end{array}$ \\
\hline Smalley et al. ${ }^{18}$ & 2006 & $\begin{array}{l}\text { 1,917 patients } \\
\text { with } \mathrm{T} 3-4, \mathrm{~N} 0, \\
\mathrm{M} 0 \text { or } \mathrm{T} 1-4, \\
\mathrm{~N} 1,2 \mathrm{M} 0 \text { rectal } \\
\text { adenocarcinoma } \\
\text { after resection }\end{array}$ & Yes-LAR or APR & $\begin{array}{l}\text { 1. Bolus 5-FU in two cycles before and } \\
\text { after RT to a dose of } 45.0 \text { Gy with a } \\
\text { minimum boost of } 5.4 \text { Gy with PVI 5-FU } \\
\text { during RT } \\
\text { 2. PVI 5-FU before, after, and } \\
\text { during radiation } \\
\text { 3. Bolus 5-FU-leucovorin-levamisole } \\
\text { before, after, and during RT }\end{array}$ & $\begin{array}{l}\text { - 3- and 5-year OS was not significantly different } \\
\text { between the three arms ( } \mathrm{p}=0.50) \\
\text { - } 3 \text { - and 5-year DFS were not significantly different } \\
\text { between the three arms ( } \mathrm{p}=0.25) \\
\text { - Grade } 3-4 \text { hematologic toxicity was much more } \\
\text { common in the two bolus FU arms: arms } 1 \text { and } 3 \\
\text { reported } 50-55 \% \text { grade } 3-4 \text { hematologic toxicity } \\
\text { versus only } 4 \% \text { in the PVI arm }\end{array}$ \\
\hline
\end{tabular}

5-FU = 5-fluorouracil; $A P R=$ abdominoperineal resection; $C R T=$ chemoradiotherapy; Gy = gray, unit of absorbed radiation dose; $L A R=$ low anterior resection; $P V I$ 5-FU = protracted venous infusion of 5-fluorouracil; $R T$ = radiotherapy. 


\section{Optimal regimen of preoperative chemotherapy}

Several trials have compared 5-FU or capecitabine-based regimens and the addition of oxaliplatin in an attempt to determine the optimal preoperative chemotherapy. In the STAR-01 trial, 747 patients with resectable locally advanced rectal cancer were randomized to receive 50.4 Gy of radiation with either infused 5-FU alone or 5-FU and oxaliplatin. ${ }^{23}$ While the pCR rate in both arms was $16 \%$ and there was no difference in the rate of APR, grade 3 and 4 toxicity was higher in the group that received oxaliplatin ( $24 \%$ versus $8 \%, p<0.001) .{ }^{23}$ Another trial that assessed the impact of adding oxaliplatin to preoperative CRT regimens was the ACCORD 12/0405 PRODIGE 2 trial. Specifically, 598 patients with locally advanced rectal cancer were randomized to receive neoadjuvant CRT with either 45 Gy and concurrent capecitabine or 50 Gy with capecitabine and oxaliplatin (CAPOX). ${ }^{24}$ Similar to the STAR-01 trial, there was no difference in PCR between the groups, but the CAPOX group experienced higher rates of grade 3 and 4 toxicity ( $25 \%$ versus $11 \%, p<0.001) .{ }^{24}$ Moreover, at 3 years, there was no significant difference in LR, OS, or DFS between the capecitabine and CAPOX groups $(6.1 \%$ versus $4.4 \%, 87.6 \%$ versus $88.3 \%$, and $67.9 \%$ versus $72.7 \%$, respectively). ${ }^{25}$ Given that the aforementioned trials demonstrated increased toxicity without benefit in clinical outcomes, oxaliplatin is not typically included in preoperative CRT.

\section{Short-course preoperative radiation}

The rationale for short-course preoperative RT in locally advanced rectal cancer is based on two major European randomized trials. The Swedish Rectal Cancer Trial randomized patients with resectable rectal cancer to receive either 25 Gy in five fractions followed by surgical resection within a week of RT, or surgery alone (Supplementary Figure 4). ${ }^{26,27}$ With a median follow-up of 13 years, patients who received preoperative RT had an significantly improved OS (38\% versus 30\%), an improved cancer-specific survival rate (72\% versus 62\%), and a decreased incidence of LR ( $9 \%$ versus $26 \%)^{26}$

The Dutch CKVO 95-04 trial also investigated the role of preoperative short-course RT, followed by TME, versus TME alone (Supplementary Figure 5). ${ }^{28}$ Of the 1,805 eligible patients, those who received RT had an decreased incidence of LR (5\% versus 11\%), but there was no difference in OS. ${ }^{29}$ However, in a subset analysis of patients with stage III cancer and negative circumferential margins, there was an improvement in OS for those who received preoperative RT ( $50 \%$ versus $40 \%, p=0.03$ ) ${ }^{29}$ Overall, these European trials established short-course radiation as a reasonable approach for preoperative therapy in rectal cancer.

\section{Preoperative short-course radiotherapy versus preoperative long-course chemoradiotherapy}

The German Rectal Cancer Trial established long-course CRT as the standard of care for patients with T3/T4 or node-positive disease, while the Swedish and Dutch trials evaluated the role of preoperative short-course RT in patients with clinical T1-3 disease. ${ }^{8,26,28}$ Subsequent trials have sought to compare these approaches. Bujko et al. randomized patients with clinical T3 rectal cancers to receive either preoperative short-course followed by TME within 7 days or CRT followed by TME within 4-6 weeks, as shown in Supplementary Figure 6. With a median follow-up of 48 months, there were no significant differences in sphincter preservation, crude LR, DFS, and OS. However, early radiation toxicity was significantly increased in the CRT group (18\% versus $3 \%, p<0.001$ ), though there was no significant difference in late toxicity or overall postoperative complications. ${ }^{30}$
The TROG Intergroup trial similarly compared preoperative short-course RT with preoperative long-course CRT, as shown in supplementary Figure 7. There were no significant differences in 3-year LR, distant recurrence, relapse-free survival, OS, or late toxicity. ${ }^{31}$ Bujko et al. also evaluated the efficacy of short-course RT followed by consolidation chemotherapy, versus long-course CRT, in improving local control in advanced rectal cancers in the Polish II trial (Supplementary Figure 8). ${ }^{32}$ The authors found that OS was significantly increased (73\% versus 65\%, $\mathrm{p}=0.046$ ) and preoperative acute toxicity was significantly reduced $(75 \%$ versus $83 \%, p=0.006$ ) in the group receiving short-course RT followed by consolidation chemotherapy versus patients who received long-course CRT. There were no significant differences in DFS, RO resection rates, PCR rates, local failure, distant metastasis, and postoperative and late complications. ${ }^{32}$ Overall, these data suggest that short-course RT is at least equivalent to long-course CRT and is more cost effective, especially in countries with limited healthcare budgets.

\section{Interval between radiotherapy and surgery}

There have been several studies evaluating the impact of timing of surgery on outcomes in rectal cancer. The Stockholm III trial randomized 840 patients with resectable rectal adenocarcinoma without evidence of metastasis to either: short-course RT (25 Gy in five fractions) followed by surgery within 1 week; short-course RT followed by surgery within 4-8 weeks; or long-course RT only (50 Gy in 25 fractions) followed by surgery within 4-8 weeks. ${ }^{33}$ There were no significant differences in LR or postoperative complications between the three groups. However, in a subgroup analysis of the two short-course arms of the study, the risk of postoperative complications was significantly lower in the patients who received short-course RT followed by a delay in surgery ( $41 \%$ versus $53 \%, p=0.001) .33$

While these data suggest that short-course radiation with a delay in surgery is an effective treatment option, these results are difficult to interpret given that the use of neoadjuvant chemotherapy was not reported, less than 20\% of patients received adjuvant chemotherapy, the long-course arm did not include concurrent chemotherapy, and centers were allowed to choose to enroll patients on only the short-course arm of the trial.

The GRECCAR-6 trial also evaluated the impact of surgical timing on outcomes in patients with advanced rectal cancer. Specifically, 265 patients with clinical T3 or T4 or node-positive tumors of the middle or lower rectum who had received long-course CRT (45-50 Gy with 5-FU or capecitabine) were randomized to either surgery 7 weeks or 11 weeks after completion of CRT. ${ }^{34}$ There was no difference between the two groups in the primary endpoint of PCR rate. However, the morbidity was significantly worse in the 11 -week group ( $44.5 \%$ versus $32 \%, p=0.0404$ ) due to a significantly higher rate of medical complications (32.8\% versus $19.2 \%, p=0.0137$ ). Furthermore, patients who received surgical resection at 11 weeks also had a significantly worse quality of mesorectal resection (78.7\% versus $90 \%$ complete resection, $\mathrm{p}=0.0156){ }^{34}$ Thus, this study suggests that delaying surgery may be associated with increased morbidity and a more difficult resection, and is longer than the maximum of 8 weeks until surgery in the Stockholm III study. Further research is needed to clarify the impact of timing on outcome in advanced rectal cancer.

\section{Total neoadjuvant therapy}

As previously discussed, neoadjuvant CRT or preoperative short-course RT followed by TME have significantly reduced LR in locally advanced 
rectal cancer.8, ${ }^{86,28}$ However, these advances have not translated into improvements in distant recurrence rates, which has increased interest in the role of systemic therapy. Currently, adjuvant chemotherapy follows neoadjuvant CRT or short-course RT and TME in the treatment paradigm for locally advanced rectal cancer, but multiple trials have emerged to clarify the role of chemotherapy and support a total neoadjuvant therapy (TNT) approach in which all RT and chemotherapy are delivered preoperatively. ${ }^{35}$

\section{Chemoradiotherapy followed by neoadjuvant chemotherapy}

One approach of TNT that has been evaluated is CRT followed by neoadjuvant chemotherapy. In a trial by Zampino et al., 51 patients affected by locally advanced rectal cancer received concurrent capecitabine and RT to a dose of 50.4 Gy followed by two cycles of capecitabine until 2 weeks prior to surgery. ${ }^{36}$ With a median follow-up of 43 months, 18\% of patients had a pCR, 12\% and 30\% were down-staged in $\mathrm{T}$ and $\mathrm{N}$, respectively. Additionally, sphincter preservations rates were $62 \%$ for patients with tumors $<6 \mathrm{~cm}$ from the anal verge and $80 \%$ for the entire cohort and 5-year DFS was $85.4 \%$ (95\% confidence interval [Cl]: 75.3-95.4\%). ${ }^{36}$ Another study by Zhu et al. also demonstrated efficacy with minimal toxicity with a TNT approach. Specifically, 42 patients with locally advanced rectal cancer received concurrent CAPOX and radiation to a dose of 44 Gy followed by one cycle of capecitabine with surgery scheduled 6 weeks after completion of neoadjuvant therapy. All patients completed neoadjuvant therapy, with the majority experiencing only mild grade 1 or 2 toxicities. ${ }^{37}$ of the entire cohort, six patients achieved PCR and 38 patients received surgery. These 38 patients were divided into good responders with tumor regression grade $3-4$ and poor responders with tumor regression grade 1-2. Good responders had improved DFS and OS compared with poor responders ( $81.6 \%$ versus $16.8 \%, p=0.000 ; 83.9 \%$ versus $40.7 \%, p=0.007) .{ }^{37}$

Moreover, in a study by Gao et al., 36 patients received concurrent CRT with two cycles of CAPOX with radiation to a total dose of $50 \mathrm{~Gy}$ and one additional cycle of chemotherapy after CRT prior to surgery. ${ }^{38}$ All patients completed concurrent CRT and underwent TME with 75\% of patients undergoing an additional sphincter-sparing surgery, but two patients were unable to complete the additional cycle of chemotherapy due to grade 3 leukopenia and diarrhea. ${ }^{38} \mathrm{~A}$ pCR, near $\mathrm{PCR}$, and minimal regression was achieved in $36.1 \%, 44.4 \%, 19.5 \%$, respectively and postoperative complications developed in $19.4 \%$ of patients. ${ }^{38}$ Finally, in a phase ॥ trial by Garcia-Aguilar et al., 292 patients with locally advanced rectal cancer received neoadjuvant CRT with 5-FU and 45 Gy with a minimum boost of 5.4 Gy and were divided into four groups: group $1 \quad(n=60)$ received TME 6-8 weeks after CRT; group $2(n=67)$ received two cycles of 5-FU, leucovorin and oxaliplatin (mFOLFOX6) between CRT and TME; group $3(n=67)$ received four cycles of mFOLFOX6 between CRT and TME; and group $4(n=65)$ received six cycles of mFOLFOX6 between CRT and TME. ${ }^{39}$ Of the 259 patients who were analyzed, 18\%, 25\%, 30\%, and 38\% of patients in groups $1,2,3$, and 4 achieved a pCR, respectively $(p=0.0036$ ). Study group was independently associated with PCR with the odds ratio of group 4 at 3.49 compared with group 1 (95\% Cl: 1.39-8.75; $p=0.011)$. $^{39}$ The most common grade 3 or higher adverse events associated with the neoadjuvant administration of mFOLFOX6 across groups 2-4 were neutropenia ( $n=5$ in group 3 and $n=6$ in group 4 ) and lymphopenia ( $n=3$ in group 3 and $n=4$ in group 4). ${ }^{39}$

\section{Neoadjuvant chemotherapy followed}

\section{by chemoradiotherapy}

Another approach of TNT that has been studied in recent trials is neoadjuvant chemotherapy followed by CRT. In a study by Fernández-Martos et al., 108 patients with locally advanced rectal cancer were randomized to preoperative CRT with CAPOX, and concurrent RT to a dose of 50.4 Gy followed by TME and four cycles of postoperative CAPOX (arm $A, n=52$ ) or induction CAPOX followed by CRT and TME (arm $B, n=56$ ). ${ }^{40,41}$ The $\mathrm{pCR}$ rates were similar between arm $A$ and arm $B$ (13.5\%, 95\% Cl: 5.6-25.8\% and 14.3\%, 95\% Cl: 6.4-26.2\%, respectively). ${ }^{40}$ However, compliance with chemotherapy was lower and toxicity with postoperative chemotherapy was higher in arm A versus arm B (57\% versus $94 \%, p<0.0001$ and $54 \%$ versus $19 \%, p=0.0004$, respectively). ${ }^{40}$ With a median follow-up of 69.5 months, there were no significant differences in DFS, OS, LR, or distant metastasis. ${ }^{41}$ In the CONTRE study by Perez et al., 39 patients with locally advanced rectal cancer received eight cycles of MFOLFOX6 followed by concurrent CRT with capecitabine to a dose of 50.4 Gy and TME 6-10 weeks after CRT. ${ }^{42}$ Among the patients in the study, $89 \%$ completed the aforementioned treatment regimen and 33\% had a pCR at the time of surgery (95\% Cl:18.24-47.76\%). ${ }^{42}$ With a median follow-up of 25.5 months, median progression-free survival was not reached. Two patients had a locoregional recurrence and six patients had a distant recurrence, but overall the regimen was well-tolerated and comparable to the current standard. ${ }^{42}$

\section{Comparison of total neoadjuvant therapy approaches}

While both aforementioned TNT approaches demonstrate promising results in comparison to the current standard of care, the optimal sequencing remains unclear. A recent trial by Fokas et al. compares the approaches to each other. Specifically, 311 patients with stage II or III rectal cancer were assigned to induction chemotherapy with three cycles of 5-FU, leucovorin, and oxaliplatin before 5-FU/oxaliplatin CRT to a dose of 50.4 Gy (group A) or to consolidation chemotherapy after CRT (group B). ${ }^{43}$ Of the 306 patients analyzed, compliance with the treatment regimen was higher in group B than group A, with $97 \%, 87 \%$, and $93 \%$ receiving full-dose RT, concomitant 5-FU, and concomitant oxaliplatin versus $91 \%$, $78 \%$, and $76 \%$ respectively. ${ }^{43}$ Additionally, CRT-related toxicity was lower (27\% versus $37 \%$ ) and PCR rate was higher in group B patients than group A patients (25\% versus 17\%). ${ }^{43}$ Further studies are needed to ultimately determine which approach of TNT translates into improved oncologic outcomes. The rationale for utilizing a TNT approach includes potentially addressing micrometastases at an early stage, reversing diverting ileostomies earlier, decreasing toxicity, and increasing tumor regression. ${ }^{44,45}$ Ultimately, the ability of TNT to improve tumor regression rates may aid in optimizing selection of patients for nonoperative management.

\section{The role of magnetic resonance imaging in risk stratification of rectal cancer}

The optimal management of rectal cancer requires assessment of tumor proximity to the mesorectal fascia in order to facilitate successful surgery, given that involvement of the CRM is highly predictive of LR and OS. ${ }^{46}$ Traditionally, primary tumors have been assessed with digital rectal exam, endorectal ultrasound, and/or pelvic computed tomography. More recently, however, rectal protocol magnetic resonance imaging (MRI) has been favored as the primary modality of tumor assessment due to its prognostic implications, with several studies demonstrating its ability to predict a curative resection with decreased inter-observer variability than 
The Evolving Role of Radiotherapy in Locally Advanced Rectal Cancer and the Potential for Nonoperative Management

endorectal ultrasound. ${ }^{77,48}$ In a prospective observational study of rectal cancers, the MERCURY study group assessed 408 patients who underwent MRI of the pelvis with a body coil and a high resolution protocol prior to TME in order to evaluate the accuracy of preoperative staging to predict surgical CRMs. The study revealed that $87 \%$ of patients had a clear CRM on histopathology, defined as $\geq 1 \mathrm{~mm}$ between the tumor and the margin, and that the specificity for prediction of a clear CRM by MRI was $92 \%$. In patients for whom MRI predicted a clear CRM and who underwent TME, 94\% actually had a clear CRM with an accuracy of prediction of $88 \%{ }^{47}$ In 5 -year follow-up from this study, MRI-involved CRM was the only preoperative staging parameter that remained significant on multivariate analysis for poorer OS, DFS, and LR compared with MRI-clear CRM with rates of $42.2 \%$ versus $62.2 \%(p<0.01), 47.3 \%$ versus $67.2 \%(p<0.05)$, and $7.1 \%$ versus $20 \%$ $(p<0.05)$, respectively. ${ }^{48}$

More recently, MRI has been used to assess pCR rates after neoadjuvant therapy. In a study by Bhoday et al., the sensitivity of assessing PCR with magnetic tumor regression grade-a system established by the Magnetic Resonance Imaging in Rectal Cancer European Equivalence study group-versus endoscopically determined residual mucosal abnormality was compared in patients with rectal adenocarcinoma who received neoadjuvant CRT before surgery, with 143 patients assessed by MRI and 119 patients assessed by residual mucosal abnormality analysis. ${ }^{49}$ Sixteen patients (13.4\%) were assessed to have a PCR by residual mucosal abnormality analysis with a sensitivity of $62.5 \%$. By contrast, 18 patients (12.6\%) were assessed to have a pCR by MRI tumor regression grade analysis with a sensitivity of $94 \%$. MRI tumor regression grading was 10 times more likely to identify a pCR than clinical assessment of residual mucosal abnormality with no difference in false positive rates. ${ }^{49}$ Furthermore, studies evaluating radiomic features in predicting tumor response have also been published and are gaining increasing importance in monitoring patients due to recent efforts to pursue nonoperative management after neoadjuvant therapy.50 Lambregts et al., evaluated the utility of MRI and diffusion-weighted imaging (DWI) in diagnosing tumor regrowth in 72 patients who underwent organ preservation after CRT for rectal cancer..$^{51}$ After assessment of 440 MRIs, 12 patients were found to have developed regrowth. The sensitivity and specificity for standard MRI were $58 \%$ and $98 \%$, respectively, while the sensitivity and specificity for standard MRI with DWI were $75 \%$ and $97 \%$, respectively. Although there was an increase in sensitivity and specificity with the addition of DWI, DWI did not result in an overall improvement in diagnostic performance in terms of area under the curve. ${ }^{51}$ While further investigations regarding the utility of MRI are needed, the aforementioned evidence suggests that it could help stratify risk in patients with rectal cancer, predict success of surgical resection, and/or identify optimal candidates for organ preservation.

\section{Nonoperative management in locally advanced rectal cancer Neoadjuvant chemoradiotherapy followed by observation}

Currently, TME remains the cornerstone of treatment for rectal cancer. Early data for the nonoperative management of rectal cancer originates from retrospective studies that have evaluated patients who were poor surgical candidates and only received either chemotherapy or CRT alone. In these studies, both local control and survival rates ranged from $20-35 \% .52 .53$ However, retrospective and prospective data have emerged from several studies evaluating observation after CRT. In a series by Habr-Gama et al. in 2006, patients with resectable rectal adenocarcinoma were treated with neoadjuvant CRT and those with a complete clinical response were observed with CT scans every 6 months and compared with 21 patients (8.3\%) who had an incomplete clinical response and ultimately underwent PTONOMO resection as shown in Supplementary Figure 9. With a mean follow-up of 57 months, there was a $3 \%$ luminal recurrence rate, $4 \%$ distant metastasis rate, and $100 \%$ 5-year OS rate. ${ }^{54}$ In an update of 361 patients, the LR rate was $5 \%$ and 5 -year OS was $93 \%$ in the patients who achieved a complete clinical response. ${ }^{55}$ In their 2013 series, Habr-Gama et al. again assessed 70 patients for complete response 10 weeks after completing neoadjuvant CRT, as shown in supplementary Figure $10 .{ }^{56} \mathrm{In}$ this cohort, 47 patients (68\%) had an initial complete response and continued monitoring without surgical resection. Of these patients, eight developed regrowth within a year, four developed recurrences after a year, and 39 (57\%) sustained a complete clinical response at a median follow-up of 56 months. Of the entire cohort, $50 \%$ did not undergo surgery. ${ }^{56}$

In a study by Maas et al., 192 patients with MRI-staged cT3, CT4 or node-positive rectal cancer underwent neoadjuvant therapy and those who had a complete clinical response-defined as no residual tumor on imaging and endoscopy - were assigned to a wait-and-see policy, as shown in Supplementary Figure 11. With a mean follow-up of 25 months, only one observed patient developed a LR which was salvaged with transanal endoscopic microsurgery (TEM) and the probability of 2-year DFS and OS was $93 \%$ and $91 \%$, respectively.57 Appelt et al. also looked at the role of observation in patients with distal rectal cancers in the lower $6 \mathrm{~cm}$ of the rectum after neoadjuvant CRT, as shown in supplementary Figure 12. In their study, 51 patients with distal T2 or T3 rectal cancers received neoadjuvant CRT. ${ }^{58}$ Forty of these patients achieved a complete clinical response and were monitored, while the rest were referred for surgical resection. With a median follow-up of 23.9 months, LR at 1 year was $15.5 \%$, while sphincter preservation was $72 \%$ at 1 year and $69 \%$ at 2 years. The most common reported acute toxicity was diarrhea and late toxicity was rectal bleeding. ${ }^{.9}$

Moreover, there have been a couple of smaller single-institution studies further supporting the use of nonoperative management for rectal cancer. For example, in a study by Dalton et al., 49 patients underwent preoperative CRT for rectal cancer and 12 patients (24\%) were found to have had a complete response per MRI, as shown in Supplementary Figure $13 .{ }^{59}$ Of these 12 patients, six progressed and were subsequently referred for surgical resection, while six remained disease-free and avoided surgery with a mean follow-up of 25.5 months..$^{59}$ In a trial by Smith et al., patients with distal T2, T3, T4, or node-positive disease who received long-course CRT were reviewed. Thirty-two patients who had a clinical complete response (no palpable tumor on exam and no pathology endoscopically) underwent nonoperative management and were compared with 57 patients who had a PCR after neoadjuvant CRT and rectal resection, as shown in Supplementary Figure $14 .{ }^{60}$ In the observation group, there were six local failures with a median time to failure of 11 months. All these patients were able to be salvaged with surgical resection and remained disease-free for a median time of 17 months. Moreover, the distant DFS and OS were similar for the nonoperative and operative groups. ${ }^{.1}$

The International Wait and Watch Database is one of the largest studies of nonoperative management in rectal cancer. In this study, 
880 rectal cancer patients who achieved a complete clinical response after neoadjuvant therapy were observed, as shown in Supplementary Figure 15. With a median follow-up of 3.3 years, the 2-year local regrowth rate was $25.2 \%$ with the vast majority of regrowth occurring within the bowel wall. For patients with local regrowth, the 5-year disease specific and OS rates were $84 \%$ and $75.4 \%$, respectively, while for patients with a sustained complete clinical response the rates were $97.3 \%$ and $87.9 \%$, respectively. ${ }^{61}$ Overall, while criteria for optimal selection of candidates for nonoperative management are still being investigated, the aforementioned data indicate that observation following neoadjuvant CRT for patients with a complete clinical response is a promising alternative to surgery.

\section{Neoadjuvant chemoradiotherapy followed by limited excision}

Organ preservation with either local excision or minimally invasive surgery is another alternative to TME and has been investigated in prospective studies. In a single-arm study by Garcia-Aguilar et al., 72 patients with T2NO distal rectal cancer were treated with neoadjuvant CRT consisting of CAPOX and $45 \mathrm{~Gy}$, with a boost to $50.4 \mathrm{~Gy}$, followed by local excision. ${ }^{62}$ With a median follow-up of 56 months, three patients developed LR and five patients developed distant recurrence; six of these patients underwent salvage surgery. For the entire cohort, 3-year DFS and OS was $88 \%$ and $95 \%$, respectively. ${ }^{62}$ GRECCAR 2 was a similar trial, which evaluated the role of local excision in 145 patients with $\mathrm{T} 2$ or $\mathrm{T} 3$ lower rectal cancer who underwent neoadjuvant CRT consisting of $50 \mathrm{~Gy}$ and concomitant CAPOX. Patients with a good clinical response (defined as $<2 \mathrm{~cm}$ of residual tumor) were randomized to either local excision $(n=74)$ or TME $(n=74) .{ }^{63}$ The primary endpoint was a composite outcome of death, recurrence, morbidity, and side-effects 2 years after surgery; one or more of these composite events occurred in $56 \%$ of patients in the local excision group and $48 \%$ of the TME group ( $p=0.43$ ). Twenty-six patients in the local excision group ultimately underwent a TME. There was no significant difference between the groups in any of the components of the composite outcome, and the superiority of local excision over TME was not established. . $^{3}$

The CARTS study by Verseveld et al. further investigated the role of organ preservation in clinically staged T1-3 No distal rectal cancer by evaluating the feasibility of TEM as an alternative to TME. Specifically, 55 patients underwent neoadjuvant CRT with 50 Gy and concurrent capecitabine, and were evaluated 6-8 weeks after completion. Patients who had a T0-2 tumor after neoadjuvant therapy underwent TEM and the other patients underwent TME. ${ }^{64}$ Fifty-one patients were eligible for the study and 47 patients proceeded to TEM. With a median follow-up of 17 months, there were only four local recurrences, three of which were in patients with ypT2 and one in a patient with ypT1 disease. Ultimately, TEM enabled organ preservation for half of these patients with rectal cancer. ${ }^{64}$ Overall, these studies suggest that neoadjuvant CRT followed by local excision may be an acceptable alternative to TME, but follow-up data are limited and further studies are necessary to validate these findings.

\section{Ongoing prospective trials of nonoperative management in rectal cancer}

There are currently additional ongoing prospective trials investigating the role of nonoperative management in rectal cancer. For example, the Cancer Institute in São Paulo has an ongoing phase II trial (ClinicalTrials.gov identifier: NCT02052921) investigating surgical resection versus observation in patients with middle and distal rectal cancer who achieved a complete clinical response after neoadjuvant CRT with study completion expected in December 2020. Additionally, a phase III trial by Vrije Universiteit Medical Center (NCT02371304) compares TME versus adjuvant CRT after local excision for early stage rectal cancers, with study completion expected in January 2023. These randomized trials are ongoing and will help to elucidate the role of nonoperative management for rectal cancer.

\section{Conclusion}

The current standard therapy for locally advanced rectal cancer is neoadjuvant CRT or short-course RT followed by TME and adjuvant chemotherapy. Studies comparing short-course RT versus long-course CRT have demonstrated equivalence, but longer follow-up is still needed. $\mathrm{MRI}$ is increasingly being used to determine $\mathrm{PCR}$ following neoadjuvant therapy to either predict optimal surgical candidates or as a mechanism for monitoring patients. While TME remains the cornerstone of treatment for locally advanced rectal cancer, nonoperative management is an emerging alternative treatment paradigm for achieving comparable oncologic control with encouraging early results. Current ongoing and future studies will continue to inform the optimal treatment paradigm in patients with locally advanced rectal cancer. $\square$
1. Bray F, Ferlay J, Soerjomataram I, et al. Global cancer statistics 2018: GLOBOCAN estimates of incidence and mortality worldwide for 36 cancers in 185 countries. CA Cancer J Clin. 2018;68:394-424

2. Fazeli MS, Keramati MR. Rectal cancer: a review. Med I Islam Repub Iran. 2015:29:171.

3. Al-Sohaily S, Biankin A, Leong R, et al. Molecular pathways in colorectal cancer. J Gastroenterol Hepatol. 2012;27:1423-31.

4. McDermott FT, Hughes ES, Pihl E, et al. Local recurrence after potentially curative resection for rectal cancer in a series of 1008 patients. Br J Surg. 1985;72:34-7.

5. Pilipshen SJ, Heilweil M, Quan SH, et al. Patterns of pelvic recurrence following definitive resections of rectal cancer. Cancer. 1984;53:1354-62.

6. McCall JL, Cox MR, Wattchow DA. Analysis of local recurrence rates after surgery alone for rectal cancer. Int $\mathrm{J}$ Colorectal Dis. 1995:10:126-32.

7. Sauer R, Becker H, Hohenberger W, et al. Preoperative versus postoperative chemoradiotherapy for rectal cancer. N Engl J Med. 2004;351:1731-40.

8. Sauer R, Liersch T, Merkel S, et al. Preoperative versus postoperative chemoradiotherapy for locally advanced rectal cancer: results of the German CAO/ARO/AlO-94 randomized phase III trial after a median follow-up of 11 years $J$ Clin Oncol. 2012;30:1926-33.

9. Miles WE. A method of performing abdomino-perineal excision for carcinoma of the rectum and of the terminal portion of the pelvic colon (1908) CA Cancer I Clin. 1971:21:361-4.

10. Galler AS, Petrelli NJ, Shakamuri SP. Rectal cancer surgery: a brief history. Surg Oncol. 2011;20:223-30.

11. Perry WB, Connaughton JC. Abdominoperineal resection: how is it done and what are the results? Clin Colon Rectal Surg. 2007;20:213-20.

12. Gastrointestinal Tumor Study Group. Prolongation of the disease-free interval in surgically treated rectal carcinoma. N Engl J Med. 1985:312:1465-72.

13. Fisher $B$, Wolmark $N$, Rockette $H$, et al. Postoperative adjuvant chemotherapy or radiation therapy for rectal cancer: results from NSABP protocol R-01. J Natl Cancer Inst. 1988;80:21-9.

14. Randomised trial of surgery alone versus surgery followed by radiotherapy for mobile cancer of the rectum. Medical Research Council Rectal Cancer Working Party Lancet 1996:348:1610-4.

15. NIH consensus conference. Adjuvant therapy for patients with colon and rectal cancer. JAMA. 1990:264:1444-50.

16. O'Connell MJ, Martenson JA, Wieand HS, et al. Improving adjuvant therapy for rectal cancer by combining protracted-infusion fluorouracil with radiation therapy after curative surgery. N Eng/ J Med. 1994;331:502-7.

17. Tepper JE, O'Connell M, Niedzwiecki D, et al. Adjuvant therapy in rectal cancer: analysis of stage, sex, and local control-final report of intergroup 0114. J Clin Oncol. 2002:20:1744-50.

18. Smalley SR, Benedetti JK, Williamson SK, et al. Phase III trial of fluorouracil-based chemotherapy regimens plus radiotherapy in postoperative adjuvant rectal cancer: GI INT 0144. J Clin Oncol.
2006:24:3542-7.

19. Gérard JP, Conroy T, Bonnetain F, et al. Preoperative radiotherapy with or without concurrent fluorouracil and leucovorin in T3-4 rectal cancers: results of FFCD 9203. J Clin Oncol. 2006;24:4620-5.

20. Bosset JF, Collette L, Calais $\mathrm{G}$, et al. Chemotherapy with preoperative radiotherapy in rectal cancer. $N$ Eng/ J Med. 2006:355:1114-23.

21. Roh MS, Colangelo $L H, O^{\prime}$ Connell MJ, et al. Preoperative multimodality therapy improves disease-free survival in patients with carcinoma of the rectum: NSABP R-03. J Clin Oncol. 2009;27:5124-30

22. Sebag-Montefiore D, Stephens RJ, Steele R, et al. Preoperative radiotherapy versus selective postoperative chemoradiotherapy in patients with rectal cancer (MRC CR07 and NCIC-CTG C016): a multicentre, randomised trial. Lancet. 2009;373:811-20.

23. Aschele C, Cionini L, Lonardi S, et al. Primary tumor response to preoperative chemoradiation with or without oxaliplatin in locally advanced rectal cancer: pathologic results of the STAR-01 randomized phase III trial. J Clin Oncol. 2011;29:2773-80.

24. Gérard JP, Azria D, Gourgou-Bourgade S, et al. Comparison of two neoadjuvant chemoradiotherapy regimens for locally advanced rectal cancer: results of the phase III trial ACCORD 12/0405-Prodige 2. J Clin Oncol. 2010;28:1638-44.

25. Gérard JP, Azria D, Gourgou-Bourgade S, et al. Clinical outcome of the ACCORD 12/0405 PRODIGE 2 randomized trial in rectal cancer. J Clin Oncol. 2012;30:4558-65.

26. Folkesson J, Birgisson $H$, Pahlman $L$, et al. Swedish Rectal Cancer 
Trial: Iong lasting benefits from radiotherapy on survival and loca recurrence rate. J Clin Oncol. 2005;23:5644-50.

27. Swedish Rectal Cancer Trial. Cedermark B, Dahlberg M, et al. Improved survival with preoperative radiotherapy in resectable rectal cancer. N Eng/ J Med. 1997:336:980-7.

28. Kapiteijn E, Marijnen CA, Nagtegaal ID, et al. Preoperative radiotherapy combined with total mesorectal excision for resectable rectal cancer. N Eng/ J Med. 2001;345:638-46.

29. van Gijn W, Marijnen CA, Nagtegaal ID, et al. Preoperative radiotherapy combined with total mesorectal excision for resectable rectal cancer: 12-year follow-up of the multicentre, randomised controlled TME trial. Lancet Oncol. 2011;12:575-82.

30. Bujko K, Nowacki MP, Nasierowska-Guttmejer A, et al. Long-term results of a randomized trial comparing preoperative short-course radiotherapy with preoperative conventionally fractionated chemoradiation for rectal cancer. Br J Surg. 2006;93:1215-23.

31. Ngan SY, Burmeister B, Fisher RJ, et al. Randomized trial of short-course radiotherapy versus long-course chemoradiation comparing rates of local recurrence in patients with $\mathrm{T} 3$ rectal cancer: Trans-Tasman Radiation Oncology Group trial 01.04. J Clin Oncol. 2012;30:3827-33.

32. Bujko K, Wyrwicz L, Rutkowski A, et al. Long-course oxaliplatin-based preoperative chemoradiation versus $5 \times 5$ Gy and consolidation chemotherapy for CT4 or fixed CT3 rectal cancer: results of a randomized phase III study. Ann Oncol. 2016;27:834-42.

33. Erlandsson J, Holm T, Pettersson D, et al. Optimal fractionation of preoperative radiotherapy and timing to surgery for rectal cancer (Stockholm III): a multicentre, randomised, non-blinded, phase 3 , non-inferiority trial. Lancet Oncol. 2017:18:336-46.

34. Lefevre JH, Mineur L, Kotti S, et al. Effect of interval (7 or 11 weeks) between neoadjuvant radiochemotherapy and surgery on complete pathologic response in rectal cancer: a multicenter randomized, controlled trial (GRECCAR-6). J Clin Oncol. 2016;34:3773-80

35. Benson AB 3rd, Venook AP, Bekaii-Saab T, et al. Rectal cancer, version 2.2015. J Natl Compr Canc Netw. 2015;13:719-28.

36. Zampino MG, Magni E, Leonardi MC, et al. Capecitabine initially concomitant to radiotherapy then perioperatively administered in locally advanced rectal cancer. Int J Radiat Oncol Biol Phys. 2009;75:421-7.

37. Zhu J, Gu W, Lian P, et al. A phase II trial of neoadjuvant IMRT-based chemoradiotherapy followed by one cycle of capecitabine for stage II/III rectal adenocarcinoma. Radiat Oncol. 2013;8:130.

38. Gao YH, Zhang $X, A n X$, et al. Oxaliplatin and capecitabine concomitant with neoadjuvant radiotherapy and extended to the resting period in high risk locally advanced rectal cancer. Strahlenther Onkol. 2014;190:158-64.

39. Garcia-Aguilar J, Chow OS, Smith DD, et al. Effect of adding
mFOLFOX6 after neoadjuvant chemoradiation in locally advanced rectal cancer: a multicentre, phase 2 trial. Lancet Oncol. 2015;16:957-66.

40. Fernández-Martos C, Pericay C, Aparicio J, et al. Phase II, randomized study of concomitant chemoradiotherapy followed by surgery and adjuvant capecitabine plus oxaliplatin (CAPOX) compared with induction CAPOX followed by concomitant compared with induction CAPOX followed by concomitan chemoradiotherapy and surgery in magnetic resonance
imaging-defined, locally advanced rectal cancer: Grupo cancer de imaging-defined, locally advanced rectal cance
recto 3 study. J Clin Oncol. 2010;28:859-65.

41. Fernández-Martos $C$, Garcia-Albeniz X, Pericay C, et al Chemoradiation, surgery and adjuvant chemotherapy versus induction chemotherapy followed by chemoradiation and surgery: long-term results of the Spanish GCR-3 phase II randomized trial. Ann Oncol. 2015:26:1722-8.

42. Perez K, Safran $\mathrm{H}$, Sikov W, et al. Complete neoadjuvant treatmen for rectal cancer: the Brown University Oncology Group CONTRE study. Am J Clin Oncol. 2017;40:283-7.

43. Fokas E, Allgäuer M, Polat B, et al. Randomized phase II trial of chemoradiotherapy plus induction or consolidation chemotherapy as total neoadjuvant therapy for locally advanced rectal cancer: CAO/ARO/AIO-12. J Clin Oncol. 2019:37:3212-22.

44. Ludmir EB, Palta M, Willett CG, CZito BG. Total neoadjuvant therapy for rectal cancer: an emerging option. Cancer. 2017;123:1497-506.

45. Franke AJ, Parekh H, Starr JS, Tan SA, et al. Total neoadjuvant therapy: a shifting paradigm in locally advanced rectal cancer management. Clin Colorectal Cancer. 2018;17:1-12.

46. Birbeck KF, Macklin CP, Tiffin NJ, et al. Rates of circumferential resection margin involvement vary between surgeons and predict outcomes in rectal cancer surgery. Ann Surg. 2002;235:449-57.

47. MERCURY Study Group. Diagnostic accuracy of preoperative magnetic resonance imaging in predicting curative resection of rectal cancer: prospective observational study. BMJ. 2006;333:779.

48. Taylor FGM, Quirke P, Heald RJ, et al. Preoperative magnetic resonance imaging assessment of circumferential resection margin predicts disease-free survival and local recurrence: 5-year follow-up results of the MERCURY study. I Clin Onc. 2013;32:34-43

49. Bhoday J, Smith F, Siddiqui MR, et al. Magnetic resonance tumor regression grade and residual mucosal abnormality as predictors for pathological complete response in rectal cancer postneoadjuvant chemoradiotherapy. Dis Colon Rectum. 2016;59:925-33.

50. Nie K, Shi L, Chen Q, et al. Rectal cancer: assessment of neoadjuvant chemoradiation outcome based on radiomics of multiparametric MRI. Clin Cancer Res. 2016:22:5256-64.

51. Lambregts DM, Lahaye MJ, Heijnen LA, et al. MRI and diffusion-weighted MRI to diagnose a local tumour regrowth during long-term follow-up of rectal cancer patients treated with organ preservation after chemoradiotherapy. Eur Radiol.
2016;26:2118-25.

52. Brierley JD, Cummings BJ, Wong CS, et al. Adenocarcinoma of the rectum treated by radical external radiation therapy. Int J Radiat Oncol Biol Phys. 1995;31:255-9.

53. Gérard JP, Chapet O, Ramaioli A, Romestaing P. Long-term control of T2-T3 rectal adenocarcinoma with radiotherapy alone. Int J Radiat Oncol Biol Phys. 2002;54:142-9.

54. Habr-Gama A, Perez RO, Nadalin W, Sabbaga J, et al. Operative versus nonoperative treatment for stage 0 distal rectal cancer following chemoradiation therapy: long-term results. Ann Surg. 2004;240:711-8.

55. Habr-Gama A, Perez RO, Proscurshim I, et al. Patterns of failure and survival for nonoperative treatment of stage $c 0$ distal rectal cancer following neoadjuvant chemoradiation therapy. J Gastrointest Surg. 2006;10:1319-29.

56. Habr-Gama A, Sabbaga J, Gama-Rodrigues J, et al. Watch and wait approach following extended neoadjuvant chemoradiation for distal rectal cancer: are we getting closer to anal cancer management? Dis Colon Rectum. 2013;56:1109-17.

57. Maas M, Beets-Tan RG, Lambregts DM, et al. Wait-and-see policy for clinical complete responders after chemoradiation for rectal cancer. J Clin Oncol. 2011:29:4633-40.

58. Appelt AL, Ploen J, Harling $\mathrm{H}$, et al. High-dose chemoradiotherapy and watchful waiting for distal rectal cancer: a prospective observational study. Lancet Oncol. 2015;16:919-27.

59. Dalton RS, Velineni R, Osborne ME, et al. A single-centre experience of chemoradiotherapy for rectal cancer: is there potential for nonoperative management? Colorectal Dis. 2012;14:567-71.

60. Smith JD, Ruby JA, Goodman KA, et al. Nonoperative management of rectal cancer with complete clinical response after neoadjuvant therapy. Ann Surg. 2012;256:965-72.

61. van der Valk MJM, Hilling DE, Bastiaannet $E$, et al. Long-term outcomes of clinical complete responders after neoadjuvan treatment for rectal cancer in the International Watch \& Wait Database (IWWD): an international multicentre registry study. Lancet. 2018;391:2537-45.

62. Garcia-Aguilar J, Renfro LA, Chow OS, et al. Organ preservation for clinical T2NO distal rectal cancer using neoadjuvant chemoradiotherapy and local excision (ACOSOG Z6041): results of an open-label, single-arm, multi-institutional, phase 2 trial. Lancet Oncol. 2015;16:1537-46.

63. Rullier E, Rouanet P, Tuech JJ, et al. Organ preservation for recta cancer (GRECCAR 2): a prospective, randomised, open-label, multicentre, phase 3 trial. Lancet. 2017;390:469-79.

64. Verseveld M, de Graaf EJ, Verhoef $\mathrm{C}$, et al. Chemoradiation therapy for rectal cancer in the distal rectum followed by organ-sparing transanal endoscopic microsurgery (CARTS study). Br J Surg. 2015:102:853-60. 